\title{
Forced Oscillation Technique: a surrogate measure of lung function in neuromuscular disease patients?
}

\author{
Esther Veldhoen ${ }^{1}$, Hans Roos ${ }^{1}$, Rolien Bekkema ${ }^{1}$, Ludo van der Pol$^{2}$, Marcel Tinnevelt ${ }^{1}$, \\ Laura Verweij-van den Oudenrijn ${ }^{3}$, Roelie Wösten-van Asperen ${ }^{3}$, Erik H.J. Hulzebos ${ }^{4}$, \\ Camiel Wijngaarde ${ }^{2}$, and Cornelis van der Ent ${ }^{3}$ \\ ${ }^{1}$ Wilhelmina Children's Hospital \\ ${ }^{2}$ UMC Utrecht Brain Center Rudolf Magnus \\ ${ }^{3}$ UMC Utrecht - Locatie WKZ \\ ${ }^{4}$ University Medical Center Utrecht
}

January 9, 2022

\begin{abstract}
Introduction Spirometry plays an important role in the assessment of possible respiratory failure in children with neuromuscular disorders (NMDs). However, obtaining reliable spirometry results is a major challenge. We studied the relation between Forced Oscillation Technique (FOT) and spirometry results. FOT is an easy, non-invasive method to measure respiratory mechanics, i.e. respiratory resistance $\mathrm{R}$ and respiratory reactance $\mathrm{X}$. We hypothesized an increased resistance $\mathrm{R}$ and reduced reactance $\mathrm{X}$ in patients with more reduced lung function. Methods In this prospective single center study we included all children with NMDs able to perform spirometry. We consecutively measured respiratory resistance $\mathrm{R}$ and reactance $\mathrm{X}$ at $5,11 \mathrm{and} 19 \mathrm{~Hz}$ and (Forced) Vital Capacity, Peak Expiratory Flow. Spearman correlation coefficients were calculated and regression curves were estimated. Results We included 148 patients, with a median age of 13 years (IQR 8-16). All correlation coefficients were statistically significant with $\mathrm{p}=0.000$. A negative correlation was found between resistance $\mathrm{R}$ and spirometry outcomes (spearman correlation coefficient $(\rho)$ between -0.5 and -0.6). A positive correlation was found between reactance X (i.e. less negative outcomes) and spirometry outcomes ( $\rho$ between 0.4 and 0.6 ). Highest correlation was found at lower frequencies. Regression analysis showed a non-linear relation between FOT and spirometry results. Conclusion We found a non-linear relation between FOT and spirometry results with increased resistance $\mathrm{R}$ and reduced reactance $\mathrm{X}$ in patients with more restrictive lung function decline. Given the difficulties with performing spirometry, FOT may be a promising surrogate measure of lung function.
\end{abstract}

Forced Oscillation Technique: a surrogate measure of lung function in neuromuscular disease patients?

Esther S. Veldhoen, MRCPCH${ }^{1}$, Johan H. Roos, $\mathrm{Msc}^{1}$, Rolien Bekkema, $\mathrm{MSc}^{2}$, W. Ludo van der Pol, $\mathrm{PhD}^{3}$ , Marcel H.B. Tinnevelt, MSc ${ }^{1}$, Laura P. Verweij-van den Oudenrijn, $\mathrm{MSc}^{1}$, Roelie M. Wösten-van Asperen, $\mathrm{PhD}^{4}$, Erik H.J. Hulzebos, $\mathrm{PhD}^{5}$, Camiel A. Wijngaarde, $\mathrm{PhD}^{3}$, C. Kors van der Ent, $\mathrm{PhD}^{2}$

${ }^{1}$ Pediatric Intensive Care Unit and Center of Home Mechanical Ventilation, Wilhelmina Children's Hospital, University Medical Center Utrecht, Utrecht University, The Netherlands

${ }^{2}$ Department of Pediatric Pulmonology, Wilhelmina Children's Hospital, University Medical Center Utrecht University, The Netherlands

${ }^{3}$ Department of Neurology, Brain Centre Rudolf Magnus, University Medical Center Utrecht, Utrecht University, The Netherlands 
${ }^{4}$ Pediatric Intensive Care Unit, Wilhelmina Children's Hospital, University Medical Center Utrecht.

${ }^{5}$ Child Development and Exercise Center, Wilhelmina Children's Hospital, University Medical Center Utrecht University, The Netherlands

Corresponding Author: E.S. Veldhoen Wilhelmina Children's Hospital, University Medical Center Utrecht, PO box 85090, 3508 AB Utrecht The Netherlands Tel +31 887554702 E.S.Veldhoen@umcutrecht.nl

No financial support.

Not presented at scientific meeting

Keywords: Neuromuscular diseases, Child, Lung function,

Abbreviated title: Forced Oscillation Technique in neuromuscular patients

Abstract

Introduction

Spirometry plays an important role in the assessment of possible respiratory failure in children with neuromuscular disorders (NMDs). However, obtaining reliable spirometry results is a major challenge. We studied the relation between Forced Oscillation Technique (FOT) and spirometry results. FOT is an easy, noninvasive method to measure respiratory mechanics, i.e. respiratory resistance $\mathrm{R}$ and respiratory reactance $\mathrm{X}$. We hypothesized an increased resistance $\mathrm{R}$ and reduced reactance $\mathrm{X}$ in patients with more reduced lung function.

Methods

In this prospective single center study we included all children with NMDs able to perform spirometry. We consecutively measured respiratory resistance $\mathrm{R}$ and reactance $\mathrm{X}$ at 5,11 and $19 \mathrm{~Hz}$ and (Forced) Vital Capacity, Peak Expiratory Flow. Spearman correlation coefficients were calculated and regression curves were estimated.

Results

We included 148 patients, with a median age of 13 years (IQR 8-16). All correlation coefficients were statistically significant with $\mathrm{p}=0.000$. A negative correlation was found between resistance $\mathrm{R}$ and spirometry outcomes (spearman correlation coefficient $(\rho)$ between -0.5 and -0.6). A positive correlation was found between reactance X (i.e. less negative outcomes) and spirometry outcomes ( $\rho$ between 0.4 and 0.6 ). Highest correlation was found at lower frequencies. Regression analysis showed a non-linear relation between FOT and spirometry results.

Conclusion

We found a non-linear relation between FOT and spirometry results with increased resistance $\mathrm{R}$ and reduced reactance $\mathrm{X}$ in patients with more restrictive lung function decline. Given the difficulties with performing spirometry, FOT may be a promising surrogate measure of lung function.

Introduction

Children with neuromuscular diseases (NMDs) may develop progressive respiratory failure due to respiratory muscle weakness. This leads to a decline in lung function, which is often further aggravated by a scoliosis and recurrent respiratory tract infections. Timing of respiratory failure varies in different NMDs ${ }^{1}$.

Guidelines suggest to measure vital capacity (VC) in all patients with NMDs who are capable of performing spirometry as part of the respiratory assessment ${ }^{2}$.

In children with NMDs spirometry results may steer clinical decision making with regards to respiratory care, support counseling about timing of pending chronic respiratory failure, and may assist in the evaluation of effects of NMD-specific treatments (such as the recently introduced survival motor neuron protein augmenting 
therapies in spinal muscular atrophy (SMA)) ${ }^{1-4}$. In healthy children reproducible spirometry is generally achievable from 6 years of age. In children of all ages who are weak, obtaining reliable spirometry results may be challenging, especially forced maneuvers ${ }^{2}$.

Respiratory function in children with NMDs is often impaired by the age at which spirometry is feasible ${ }^{5}$. For this reason, there is an urgent need for alternative noninvasive lung function tests in children unable to perform spirometry ${ }^{1}$. Forced Oscillation Technique (FOT) may serve as a surrogate test in children unable to perform spirometry. FOT is a noninvasive, versatile method to measure respiratory mechanics. FOT can be performed in less than half a minute ${ }^{6}$. Small-amplitude pressure oscillations are superimposed on the normal breathing, thereby avoiding the need for any special breathing maneuver or any noticeable interference with respiration ${ }^{7}$. FOT measures respiratory resistance $\mathrm{R}$ and respiratory reactance $\mathrm{X}$. Resistance $\mathrm{R}$ describes the dissipative mechanical properties of the respiratory system, and mainly measures viscous resistance in large airways and to a lesser extent the tissue resistance. Reactance X measures the relationship between pressure and volume (the elastic properties) at low oscillation frequencies and the relationship between pressure and volume acceleration (the inertive properties) which become progressively more important at increasing frequencies ${ }^{5,7}$. Previous studies have shown that FOT is feasible in children with SMA as young as 3 years ${ }^{5,8}$. Here, we aimed to assess the relation between results of FOT and spirometry in children with NMDs and hypothesized the presence of a correlation between these tests. We hypothesized an increased resistance $\mathrm{R}$ and reduced reactance $\mathrm{X}$ and in patients with more restrictive lung function decline, due to reduced compliance of the chest wall and reduced lung volumes ${ }^{5}$.

Methods

In this prospective cohort study, all children with NMDs attending the outpatient department of the Center of Home Mechanical Ventilation, and able to perform spirometry were included once between August 2019 and May 2021. Patients with tracheostomy were excluded.

FOT (ResmonPro Restech $囚$ ) and spirometry data (Geratherm Spirostik ${ }^{\circledR}$ ) were measured consecutively at the department of pediatric pulmonology at our hospital. These lung function tests were performed by a small team of professionals experienced in conducting these tests in children.

To measure FOT, children were seated with the head in neutral position, connected to the oscillation device via a mouthpiece and a noseclip in place. Cheek and floor of mouth were supported by the lung function technician. Measurements were obtained according to the American Thoracic Society/European Respiratory Society guidelines ${ }^{9}$. FOT was measured at $5 \mathrm{~Hz}, 11 \mathrm{~Hz}$, and $19 \mathrm{~Hz}$. We studied (Forced) Vital Capacity $((\mathrm{F}) \mathrm{VC})$ and Peak Expiratory Flow (PEF). Spirometry was measured and reported according to the European Respiratory Society guidelines ${ }^{10}$. All tests were performed in sitting position, without corsets or braces.

We studied the relation between resistance $\mathrm{R}$ and reactance $\mathrm{X}$ during inspiration (insp), expiration (exp) and total breath (tot) and (F)VC and PEF. We did not study standardized FOT results, as standardized values are not available at these frequencies in children.

The study was approved by the institutional medical ethical committee.

Statistical analysis

We used descriptive statistics to describe baseline characteristics. Only measurements obtained during the first visit after inclusion were used for analyses. We calculated the non-parametric two-tailed spearman correlation coefficients $(\rho)$ to describe the relation between FOT and spirometry results. Regression curve was estimated using IBM SPSS 26.0.

Results

We included 148 patients with a median age of 13 years. One third of the patients were patients with spinal muscular atrophy (SMA), one quarter of the patients were patients with Duchenne Muscular Atrophy (DMD). 
Fifteen percent of the patients were supported by home mechanical ventilation. Baseline characteristics are shown in Table 1.

FOT measurement was feasible in all children. Spearman correlation coefficients of the relation between FOT and spirometry results are shown in Table 2. All correlation coefficients were statistically significant with $\mathrm{p}=0.000$. A negative correlation was found between resistance $\mathrm{R}$ and spirometry outcomes (spearman correlation coefficient $(\rho)$ between -0.5 and -0.6 ). A positive correlation was found between reactance X (i.e. less negative outcomes) and spirometry outcomes ( $\rho$ between 0.4 and 0.6 ). Highest correlation was found at lower frequencies. This confirmed our hypothesis: an increased resistance $\mathrm{R}$ and reduced reactance $\mathrm{X}$ was observed in patients with lower (F)VC and PEF.

Regression curves showing the relation between both respiratory resistance $\mathrm{R}$ and reactance $\mathrm{X}$ measured at $5 \mathrm{~Hz}$ and spirometry results are shown in respectively Figure 1 and Figure 2.

\section{Discussion}

In this study we have shown that FOT may be used as a surrogate measure of lung function in children with NMDs, especially FOT measurements at lower frequencies. An increased resistance $\mathrm{R}$ and reduced reactance $\mathrm{X}$ was observed in patients with lower (F)VC and PEF.

Although obtaining reliable spirometry results are challenging in young or severely affected patients with NMDs, spirometry results are important outcomes in patients with NMDs. For example, in these patients significantly lowered FVC values are associated with an increased risk of REM- and NREM-related sleep disordered breathing ${ }^{2,11}$, and PEF has been shown to be a sensitive marker to monitor respiratory muscle strength in patients with $\mathrm{DMD}^{12}$. However, limited data are available on FOT in patients with NMDs. Although FOT provides an objective measure, it does not measure the same aspects of respiratory function as other tests ${ }^{5}$. Respiratory resistance $\mathrm{R}$ mainly measures viscous resistance in large airways and, to a lesser extent, tissue resistance. In patients with NMDs tissue compliance is reduced due to underinflation and microatelectasis. As compliance reduces, it is expected that tissue resistance and R values increase ${ }^{5}$. In this study we confirmed increased $\mathrm{R}$ values with decreased spirometry results. In patients with NMDs the reactance $\mathrm{X}$ is expected to be reduced due to reduced compliance of the chest wall and reduced lung volumes $^{5}$. In this study we confirmed more reduced, i.e. more negative, $\mathrm{X}$ values in patients with more reduced spirometry outcomes.

Two previous studies by the same group have shown that the use of FOT as a surrogate outcome to measure lung function was feasible in children with SMA. Gauld, et al. showed a linear relationship between respiratory reactance $\mathrm{X}$ measured at $8 \mathrm{~Hz}$ and $\mathrm{FVC}$ in 4 children with $\mathrm{SMA}^{5}$. Kapur, et al. showed that children with SMA requiring non-invasive ventilation (NIV) $(n=10)$ had an abnormal respiratory resistance $\mathrm{R}$ measured at $8 \mathrm{~Hz}$ compared to children not using NIV $(\mathrm{n}=15)^{8}$. Although not linear, we confirmed the significant relation between spirometry and resistance $\mathrm{R}$ and reactance $\mathrm{X}$ in this larger cohort.

Our study has several limitations. Firstly, in contrast to previous studies ${ }^{5,8}$ we did not study standardized values of FOT. To our knowledge, no pediatric reference values are available of the frequencies used in this study . Secondly, as this study was not aimed at studying feasibility of FOT measurements in young or less cooperative children, we only included patients able to perform spirometry. It therefore remains unclear whether our results are generalizable to much younger children with NMDs, although we expect this to be the case as FOT has been shown to allow for evaluations of respiratory mechanics in neonates and very young children. FOT is able to distinguish between healthy neonates and neonates with respiratory problems ${ }^{6}$.

Although the number of included patients in this study are more than 10 times higher than in previous studies, we did not study follow up data of these patients. Research is required to include repeated measures over time, to study the ability to predict lung function decline by using FOT.

Conclusion

We observed a non-linear relation between FOT measurements and spirometry results in children with NMDs. 
We confirmed our hypothesis and found an increased resistance $\mathrm{R}$ and reduced reactance $\mathrm{X}$ in patients with more restrictive lung function decline. FOT may be promising as a surrogate measure of lung function in these children.

References

1. Fauroux B, Khirani S. Neuromuscular disease and respiratory physiology in children: Putting lung function into perspective. Respirology 2014;19(6):782-791.

2. Hull J, Aniapravan R, Chan E, Chatwin M, Forton J, Gallagher J, Gibson N, Gordon J, Hughes I, McCulloch R, et al. Guideline for respiratory management of children with neuromuscular disease. Thorax 2012;67:1-40.

3. Buu MC. Respiratory complications, management and treatments for neuromuscular disease in children. Curr Opin Pediatr 2017;29:326-333.

4. Gower WA, Birnkrant DJ, Black JB, Noah TL. Pediatric Pulmonology Year in Review 2018: Rare lung disease, neuromuscular disease, and diagnostic testing. Pediatr Pulmonol 2019;54:1655-1662.

5. Gauld LM, Keeling LA, Shackleton CE, Sly PD. Forced oscillation technique in spinal muscular atrophy. Chest 2014;146(3):795-803.

6. Klinger AP, Travers CP, Martin A, Kuo HC, Alishlash AS, Harris WT, Carlo WA, Ambalavanan N. Noninvasive forced oscillometry to quantify respiratory mechanics in term neonates. Pediatr Res 2020;88(2):293299.

7. Oostveen E, Macleod D, Lorino H, Farré R, Hantos Z, Desager K, Marchal F. The forced oscillation technique in clinical practice: methodology, recommendations and future developments on behalf of the ERS Task Force on Respiratory Impedance Measurements. Eur Respir J 2003;22(6):1026-1041.

8. Kapur N, Deegan S, Parakh A, Gauld L. Relationship between respiratory function and need for NIV in childhood SMA. Pediatr Pulmonol 2019;54(11):1174-1180.

9. Beydon N, Davis SD, Lombardi E, Allen JL, Arets HGM, Aurora P, Bisgaard H, Davis GM, Ducharme FM, Eigen H, et al. An Official American Thoracic Society/European Respiratory Society Statement: Pulmonary function testing in preschool children. Am J Respir Crit Care Med 2007;175(12):1304-1345.

10. Miller MR, Hankinson J, Brusasco V, Burgos F, Casaburi R, Coates A, Crapo R, Enright P, van der Grinten CPM, Gustafsson P, et al. Standardisation of spirometry. Eur Respir J 2005;26(2):319-338.

11. Finkel RS, Mercuri E, Meyer OH, Simonds AK, Schroth MK, Graham RJ, Kirschner J, Iannaccone ST, Crawford TO, Woods S, et al. Diagnosis and management of spinal muscular atrophy: Part 2: Pulmonary and acute care; medications, supplements and immunizations; other organ systems; and ethics. Neuromuscul Disord 2018;28(3):197-207.

12. Buyse GM, Goemans N, Van Den Hauwe M, Meier T. Effects of glucocorticoids and idebenone on respiratory function in patients with duchenne muscular dystrophy. Pediatr Pulmonol 2013;48(9):912-920. 

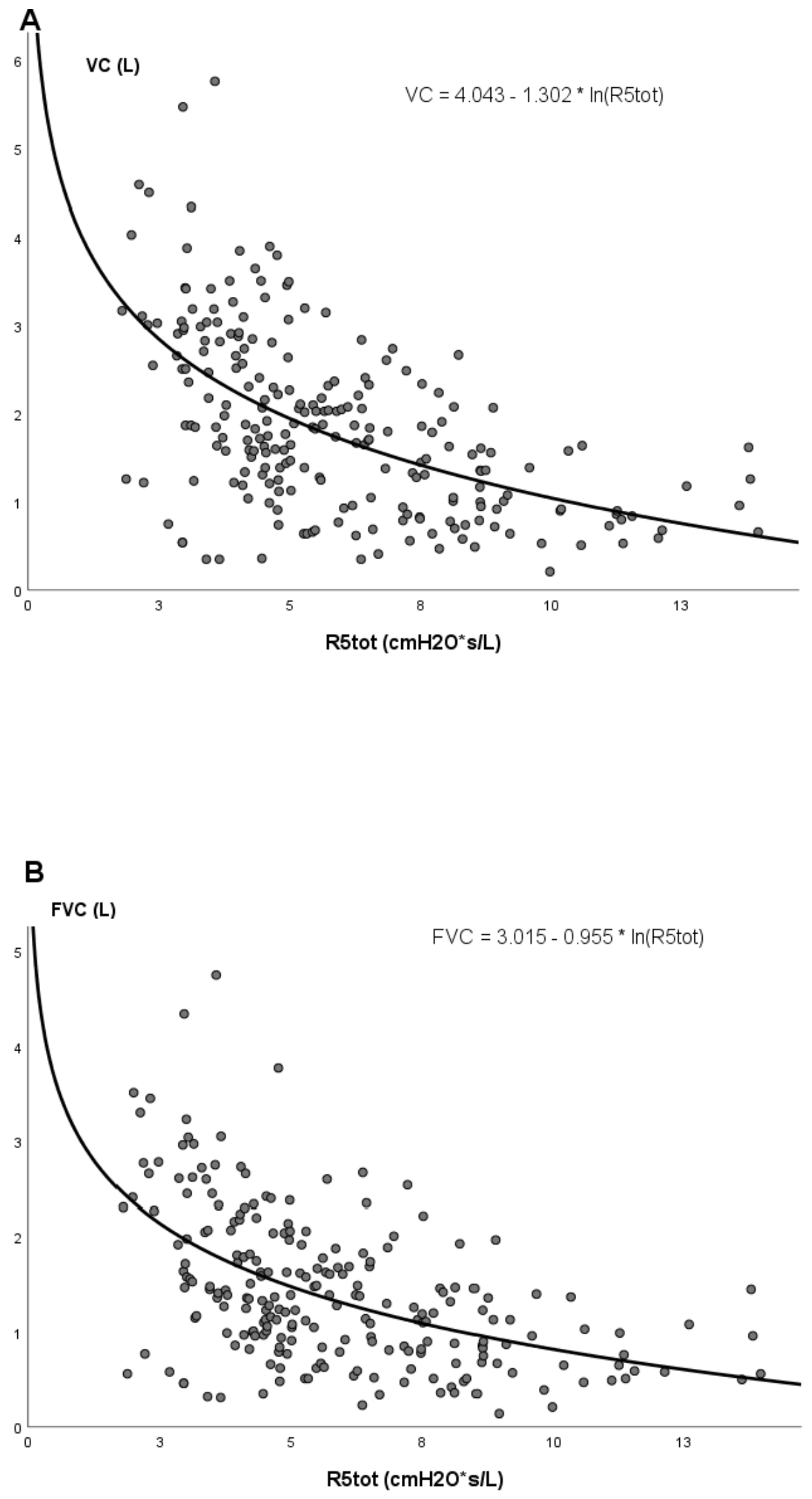


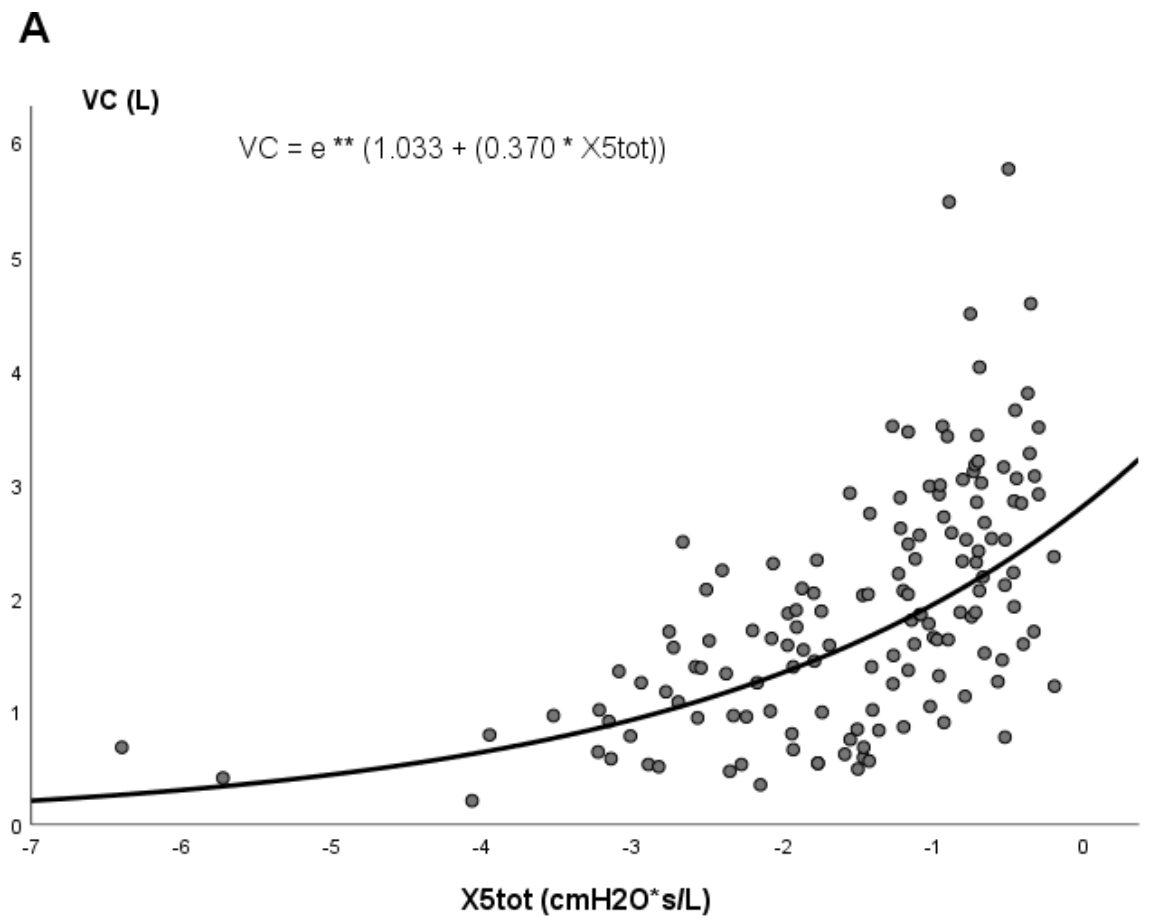

B

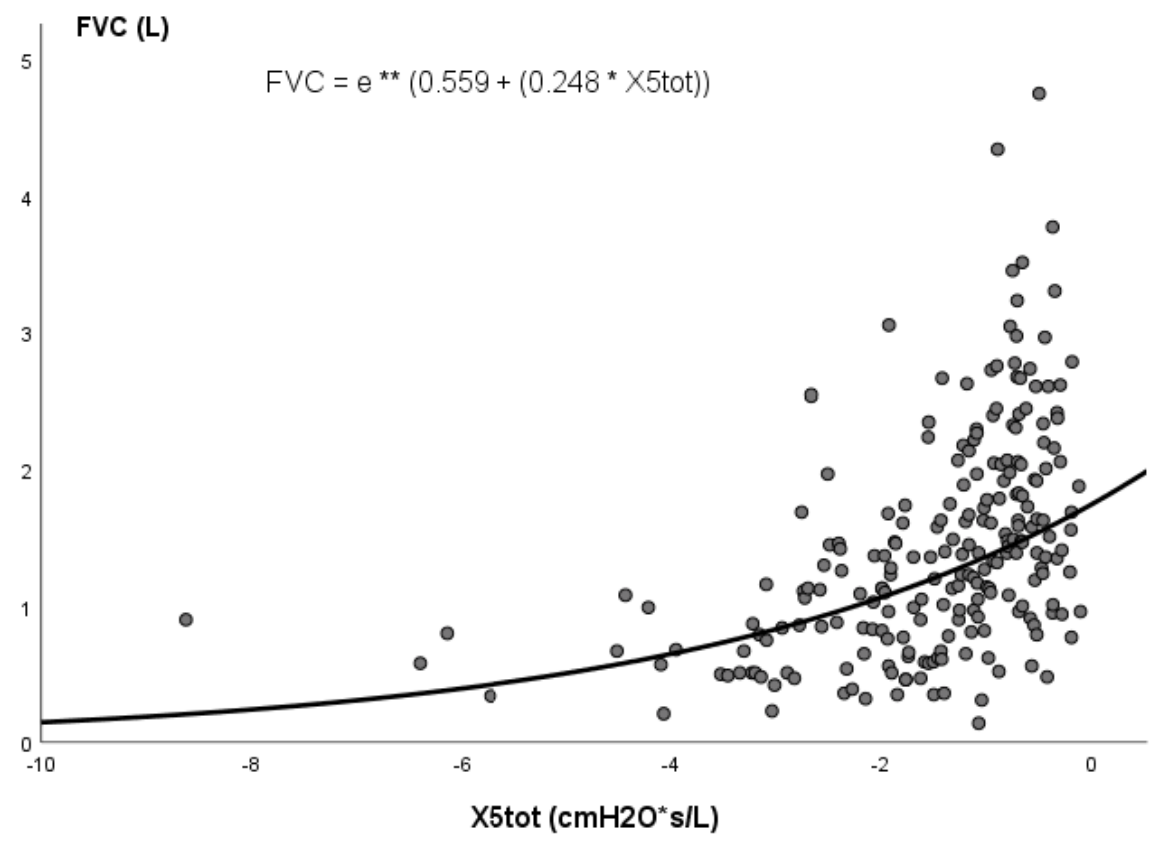




\section{Hosted file}

Table 1 Patient characteristics-0112.docx available at https://authorea.com/users/438154/ articles/552185-forced-oscillation-technique-a-surrogate-measure-of-lung-function-inneuromuscular-disease-patients

\section{Hosted file}

Table 2 Relation between FOT results and spirometry.docx available at https://authorea.com/ users/438154/articles/552185-forced-oscillation-technique-a-surrogate-measure-of-lungfunction-in-neuromuscular-disease-patients 